DiscReTE ANALYSis, 2016:10, 28 pp.

www.discreteanalysisjournal.com

\title{
Geometric Stability via Information Theory
}

\author{
David Ellis \\ Ehud Friedgut* \\ Guy Kindler \\ Amir Yehudayoff ${ }^{\dagger}$
}

Received 29 November 2015; Revised 24 June 2016; Published 28 June 2016

\begin{abstract}
The Loomis-Whitney inequality, and the more general Uniform Cover inequality, bound the volume of a body in terms of a product of the volumes of lower-dimensional projections of the body. In this paper, we prove stability versions of these inequalities, showing that when they are close to being tight, the body in question is close in symmetric difference to a box. Our results are best possible up to a constant factor depending upon the dimension alone. Our approach is information theoretic.

We use our stability result for the Loomis-Whitney inequality to obtain a stability result for the edge-isoperimetric inequality in the infinite $d$-dimensional lattice. Namely, we prove that a subset of $\mathbb{Z}^{d}$ with small edge-boundary must be close in symmetric difference to a $d$-dimensional cube. Our bound is, again, best possible up to a constant factor depending upon $d$ alone.
\end{abstract}

Key words and phrases: Loomis-Whitney inequality, projections, stability, information theory, entropy

\section{Introduction}

In this paper, we prove stability results for the Loomis-Whitney inequality and some of its generalisations. Let us start by describing these results.

\subsection{Projection inequalities}

The Loomis-Whitney inequality. The Loomis-Whitney inequality [22] bounds the volume of a $d$ dimensional body in terms of the volumes of its $(d-1)$-dimensional projections. It states that every body $S$ in $\mathbb{R}^{d}$ satisfies

$$
\mu(S)^{d-1} \leq \prod_{i \in[d]} \mu\left(\pi_{[d] \backslash\{i\}}(S)\right),
$$

\footnotetext{
* Supported by I.S.F. grant 1168/15 and by Minerva grant 712023.
}

${ }^{\dagger}$ Supported by I.S.F. grant 503/11 
where $\mu$ denotes Lebesgue measure, $[d]:=\{1,2, \ldots, d\}$, and for $g \subset[d]$, we denote by $\pi_{g}(S)$ the projection of $S$ onto the coordinates of $g$ (i.e., the projection onto the subspace $\left\{x \in \mathbb{R}^{d}: x_{i}=0 \forall i \notin g\right\}$ ). Here, we say that $S \subset \mathbb{R}^{d}$ is a body if it is an open set with compact closure. Note that if $S$ is a Cartesian product of subsets of $\mathbb{R}$, then equality holds in (1); we call such a body a box.

The Box Theorem and the Uniform Cover inequality. The Box Theorem of Bollobás and Thomason [7] is a simultaneous generalisation and strengthening of the Loomis-Whitney inequality. It states that for any body $S \subset \mathbb{R}^{d}$, there exists a box $B \subset \mathbb{R}^{d}$ with the same measure as $S$, such that $\mu\left(\pi_{g}(S)\right) \geq \mu\left(\pi_{g}(B)\right)$ for all $g \subset[d]$. Bollobás and Thomason show that this is equivalent to the so-called 'Uniform Cover inequality' of Chung, Frankl, Graham and Shearer [9]. We say that a family $\mathcal{G} \subset \mathcal{P}([d])$ is a uniform $m$-cover if every $i \in[d]$ belongs to exactly $m$ of the sets in $\mathcal{G}$, and that $\mathcal{G}$ is a uniform cover if $\mathcal{G}$ is a uniform $m$-cover for some $m \in \mathbb{N}$. The Uniform Cover inequality states that for any body $S \subset \mathbb{R}^{d}$, any $m \in \mathbb{N}$, and any uniform $m$-cover $\mathcal{G} \subset \mathcal{P}([d])$, we have

$$
\mu(S)^{m} \leq \prod_{g \in \mathcal{G}} \mu\left(\pi_{g}(S)\right) .
$$

The Uniform Cover inequality is sharp when $S$ is a box.

Applying the Uniform Cover inequality to sets which are unions of axis-parallel unit cubes, implies that for any finite $S \subset \mathbb{Z}^{d}$, any $m \in \mathbb{N}$, and any uniform $m$-cover $\mathcal{G} \subset \mathcal{P}([d])$, we have

$$
|S|^{m} \leq \prod_{g \in \mathcal{G}}\left|\pi_{g}(S)\right|
$$

(Here, of course, $|S|$ denotes the cardinality of the set $S$.) Since increasing the size of a set $g \in \mathcal{G}$ can only increase the right-hand side of (3), it follows that for any set family $\mathcal{G} \subset \mathcal{P}([d])$, and any finite $S \subset \mathbb{Z}^{d}$, we have

$$
|S|^{m(\mathcal{G})} \leq \prod_{g \in \mathcal{G}}\left|\pi_{g}(S)\right|
$$

where $m(\mathcal{G})$ denotes the minimum integer $m$ such that every $i \in[d]$ belongs to at least $m$ of the sets in $\mathcal{G}$.

In fact, (3) implies (2), by a standard approximation argument, approximating a body $S \subset \mathbb{R}^{d}$ by a union of cubes in a sufficiently fine grid, as outlined in [22]. Note, however, that the analogue of (4) for bodies in $\mathbb{R}^{d}$ (with Lebesgue measure) does not necessarily hold if $\mathcal{G}$ is not a uniform cover, as can be seen by taking $S$ to be a $d$-dimensional axis-parallel cube of side-length less than 1. (Roughly speaking, the approximation argument requires both sides of (2) to scale by the same factor, when $S$ is dilated; see the proof of Corollary 2 in Section 3.1 below.)

Shearer's Lemma. Loomis and Whitney, and Bollobás and Thomason, proved their results using induction on the dimension, and Hölder's inequality. However, the discrete versions of the LoomisWhitney and Uniform Cover inequalities (which are equivalent to the continuous ones) are special cases of Shearer's Entropy Lemma. The term 'Shearer's Lemma' is often used to refer to two essentially equivalent results. The first is stated in terms of the entropy of a random variable, first proved by Shearer (implicitly) in 1978, and first published by Chung, Frankl, Graham and Shearer in 1986 in [9]. The 


\section{GeOMETRic Stability Via Information TheORY}

second, from the same paper, is equation (2), the result we referred to above as the Uniform Cover inequality. The entropy approach for proving these inequalities is the one we adopt in this paper.

As well as being very natural statements in their own right, the above results have many applications: for example in convex geometry (see [3]), in the study of isoperimetric problems (see [22]), in extremal combinatorics (see [9]) and in additive combinatorics (see [1]). There are also several useful generalisations of Shearer's Lemma, such as the weighted version in [19], which is itself a special case of the Brascamp-Lieb inequality [8]. In [2], Ball stated and applied the geometric version of the Brascamp-Lieb inequality to study sections of the Euclidean cube. The results in this paper apply directly to several of these generalisations.

\section{Stability versions}

When a geometric, combinatorial or functional inequality is sharp, it is natural to ask whether it is also 'stable' - i.e., when the inequality is almost sharp for a particular object, must that object be close in structure to an extremal one (meaning, an object for which equality holds)?

Stability phenomena for geometric and functional inequalities have been widely studied in recent years. To obtain a stability result for an inequality, it is natural to look closely at known proofs of the inequality and see what information these proofs yield about objects where equality 'almost' holds. Several methods for proving geometric inequalities have recently been shown to yield best-possible (or close to best-possible) stability results. A partial list includes symmetrization techniques (see e.g. [21]), optimal transport (see e.g. [16, 10]), spectral techniques (see e.g. [5]), and non-linear evolution equations (see e.g. [12]). Stability phenomena for combinatorial inequalities have also been widely studied, and best-possible (or close to best-possible) stability results have been obtained via elementary combinatorial arguments (see e.g. [26, 17]), and using spectral techniques (see e.g. [20, 13]), Fourier analysis (see e.g. $[20,18]$ ) and 'non-Abelian' Fourier analysis (see e.g. $[13,14,15]$ ).

Our main result in this paper is a stability result for the Uniform Cover inequality. To state it, we need some more notation. We define a box in $\mathbb{Z}^{d}$ to be a Cartesian product of finite subsets of $\mathbb{Z}$. For a collection of subsets of coordinates $\mathcal{G} \subset \mathcal{P}([d])$, we denote by $\sigma(\mathcal{G})$ the maximum integer $\sigma$ such that for every $i, j \in[d]$ with $i \neq j$, there are at least $\sigma$ sets in $\mathcal{G}$ containing $i$ but not $j$. If $\sigma(\mathcal{G})>0$, then we define

$$
\rho(\mathcal{G}):=\frac{m(\mathcal{G})}{\sigma(\mathcal{G})} .
$$

We prove the following stability result for the inequality (4).

Theorem 1. For every integer $d \geq 2$ there exists $b=b(d)>0$ such that the following holds. Let $\mathcal{G} \subset \mathcal{P}([d])$ with $m(\mathcal{G}), \sigma(\mathcal{G})>0$. Let $S \subset \mathbb{Z}^{d}$ with $|S|<\infty$. If

$$
|S| \geq(1-\varepsilon)\left(\prod_{g \in \mathcal{G}}\left|\pi_{g}(S)\right|\right)^{1 / m(\mathcal{G})},
$$

then there exists a box $B \subset \mathbb{Z}^{d}$ such that

$$
|S \Delta B| \leq b \rho(\mathcal{G}) \varepsilon|S| .
$$


Our proof yields $b(d)=4 d^{2}+64 d$. This theorem is best-possible in terms of its dependence upon $\varepsilon$, as can be seen by taking $S=[a]^{d} \backslash\left[a^{\prime}\right]^{d}$, where $\left(a^{\prime} / a\right)^{d}=\varepsilon<2^{-d}$, and taking $\mathcal{G}=[d]^{(d-1)}$.

Theorem 1 easily implies the following analogue for bodies (with uniform covers), via the standard approximation argument outlined in [22], and referred to above.

Corollary 2. For every integer $d \geq 2$ there exists $b=b(d)>0$ such that the following holds. Let $m \in \mathbb{N}$, and let $\mathcal{G} \subset \mathcal{P}([d])$ be a uniform $m$-cover with $\sigma(\mathcal{G})>0$. Let $S \subset \mathbb{R}^{d}$ be a body such that

$$
\mu(S) \geq(1-\varepsilon)\left(\prod_{g \in \mathcal{G}} \mu\left(\pi_{g}(S)\right)\right)^{1 / m} .
$$

Then there exists a box $B \subset \mathbb{R}^{d}$ such that

$$
\mu(S \Delta B) \leq b \rho(\mathcal{G}) \varepsilon \mu(S) .
$$

For completeness, we present in full the deduction of Corollary 2 from Theorem 1, in Section 3.1.

If $\mathcal{G}=[d]^{(d-1)}$, then $m(\mathcal{G})=d-1, \sigma(\mathcal{G})=1$ and $\rho(\mathcal{G})=d-1$, so the following stability result for the Loomis-Whitney inequality, is a special case of Theorem 1 .

Corollary 3. For every integer $d \geq 2$ there exists $c=c(d)>0$ such that the following holds. Let $S \subset \mathbb{Z}^{d}$ with $|S|<\infty$. If

$$
|S| \geq(1-\varepsilon)\left(\prod_{i=1}^{d}\left|\pi_{[d] \backslash\{i\}}(S)\right|\right)^{1 /(d-1)},
$$

then there exists a box $B \subset \mathbb{Z}^{d}$ such that

$$
|S \Delta B| \leq c \varepsilon|S|
$$

Of course, we can take $c(d)=(d-1) b(d)=(d-1)\left(4 d^{2}+64 d\right) \leq 36 d^{3}$ in this corollary.

Stability for a weighted version of the Uniform Cover inequality. In [19], a weighted version of the Uniform Cover inequality is proved, a version that is, in fact, a special case of the Brascamp-Lieb inequality of [8]. It is not hard to verify that our proof in this paper goes through almost word for word to yield the following stability result for the weighted version.

Theorem 4. For every integer $d \geq 2$ there exists $b=b(d)>0$ such that the following holds. Let $\mathcal{G} \subset \mathcal{P}([d])$, and let $w: \mathcal{G} \rightarrow \mathbb{R}_{\geq 0}$ be a non-negative weight function on $\mathcal{G}$, such that every $i \in[d]$ is covered by sets with total weight at least 1, i.e.

$$
\sum_{\substack{g \in \mathcal{G} \\ i \in g}} w(g) \geq 1
$$

Let $\sigma(\mathcal{G}):=\min _{i \neq j} \sum_{g \cap\{i, j\}=\{i\}} w(g)$, assume $\sigma(\mathcal{G})>0$, and let $\rho(\mathcal{G})=1 / \sigma(\mathcal{G})$. Let $S \subset \mathbb{Z}^{d}$ with $|S|<\infty$. If

$$
|S| \geq(1-\varepsilon) \prod_{g \in \mathcal{G}}\left|\pi_{g}(S)\right|^{w(g)}
$$




\title{
GEOMETRIC STABILITY VIA INFORMATION THEORY
}

then there exists a box $B \subset \mathbb{Z}^{d}$ such that

$$
|S \Delta B| \leq b \rho(\mathcal{G}) \varepsilon|S|
$$

Again, we can take $b(d)=4 d^{2}+64 d$. We omit the details of the proof of Theorem 4 .

\begin{abstract}
About the proof. A few words regarding our proof of Theorem 1. To prove a stability result for some inequality, it is natural to consider a certain proof of that inequality, and to 'work backwards' through this proof to deduce closeness to the desired structure when equality almost holds. This may be called a 'stable version' of the relevant proof. Perhaps the first natural approach to proving Theorem 1 is to produce a stable version of the classical proof of (4), which uses Hölder's inequality and induction on the dimension. However, even for the Loomis-Whitney inequality, this only yields $\Theta(\sqrt{\varepsilon})$-dependence. In order to obtain the sharp $\Theta(\varepsilon)$-dependence we seek, we consider the beautiful Llewellyn-Radhakrishnan proof of Shearer's Lemma (see [24]). This proof is information-theoretic, using simple properties of entropy.
\end{abstract}

Given a set $S \subset \mathbb{Z}^{d}$ and a family $\mathcal{G} \subset \mathcal{P}([d])$, such that the inequality (4) is almost tight for $S$ and $\mathcal{G}$, we first deduce that, in a sense, $S$ is 'close' to a 'product structure'; that is, the uniform distribution on $S$ is 'close' to the product of its marginals (the relevant notion of distance is discussed below). That part of the argument is a fairly straightforward deduction from the entropy proof of Llewellyn-Radhakrishnan, or alternatively from the entropy inequalities proved by Balister and Bollobás in [1]. (It is also inspired by the proof of the Parallel Repetition Theorem in [25], and related works such as [4].)

Although information theory allows us to easily identify a product structure, it moves us from the language of sets to the language of distributions. The next ingredient of the proof is more combinatorial. Its purpose is to move back from the language of distributions to the language of sets; we identify the actual box that we claim exists.

It turns out that for this part of the proof, a 'two-dimensional lemma' suffices. Given a set $S \subset X_{1} \times X_{2}$, such that the uniform distribution on $S$ is 'close' to the product of its marginals, we find a two-dimensional box (a 'rectangle') $R_{1} \times R_{2}$, which is a good approximation of the set $S$. To prove this lemma, we need to identify the two sets $R_{1} \subset \pi_{1}(S)$ and $R_{2} \subset \pi_{2}(S)$. This is done via an iterative 'trimming' procedure, which gradually removes parts of $\pi_{1}(S)$ and $\pi_{2}(S)$, until only $R_{1}$ and $R_{2}$ remain. The crux of the proof is in showing that we did not throw too much, i.e., that $S \backslash\left(R_{1} \times R_{2}\right)$ is small.

We apply this two-dimensional lemma $d$ times; for each $i \in[d]$ we consider our set $S \subset \mathbb{Z}^{d}$ as a two-dimensional set $S \subset \mathbb{Z}^{\{i\}} \times \mathbb{Z}^{[d] \backslash\{i\}}$, and we find a set $R_{i} \subset \mathbb{Z}^{\{i\}}$ which is a good candidate to be the 'edge in direction $i$ ' of the box approximating $S$. We then check that, indeed, $S$ is close to the box $R_{1} \times R_{2} \times \ldots \times R_{d}$.

The last issue to discuss is the meaning of the word 'close' above: how to measure the 'distance' between the uniform distribution on $S$ and the product of its marginals. The information theoretic part of the argument naturally leads to measuring this distance using the Kullback-Leibler divergence. A natural thing to do at this point would be to use Pinsker's inequality to move from Kullback-Leibler divergence to $\ell^{1}$-distance. This, however, leads to suboptimal $\Theta(\sqrt{\varepsilon})$-dependence. To overcome this difficulty, we introduce a new (but natural) measure of distance, which we call the 'hole-weight'. The hole-weight suffices to control the trimming procedure, yielding optimal $\Theta(\varepsilon)$-dependence. It may find applications in other, similar scenarios. For more details on this part of the proof, see Section 3.1 below. 


\subsection{Isoperimetric inequalities}

In Section 4, we apply Theorem 1 to prove another result, demonstrating stability for the edge-isoperimetric inequality in the infinite $d$-dimensional lattice. Before stating this formally, we give some background on isoperimetric inequalities.

Isoperimetric problems are classical objects of study in mathematics. In general, they ask for the minimum possible 'boundary size' of a set of a given 'size', where the exact meaning of these words varies according to the problem.

The classical isoperimetric problem in the plane asks for the minimum possible perimeter of a shape in the plane with area 1 . The answer, that it is best to take a circle, was already known to the ancient Greeks, but it was not until the 19th century that this was proved rigorously ${ }^{1}$.

The isoperimetric inequality for Euclidean space states that among all subsets of $\mathbb{R}^{d}$ of given volume, Euclidean balls have the smallest boundary. To state a version of this precisely, if $A \subset \mathbb{R}^{d}$ is a Borel set of finite Lebesgue measure, we denote by $\operatorname{Per}(A)$ the distributional perimeter of $A$ (see e.g. Chapter 12 in [23] for the definition of distributional perimeter). When $A$ has piecewise smooth topological boundary $\partial A$, then $\operatorname{Per}(A)=\mu_{d-1}(\partial A)$, where $\mu_{d-1}(\partial A)$ denotes the $(d-1)$-dimensional Hausdorff measure of $\partial A$, a measure of the boundary which may be more familiar to some readers.

Theorem 5. If $S \subset \mathbb{R}^{d}$ is a Borel set with Lebesgue measure $\mu(S)<\infty$, then

$$
\operatorname{Per}(S) \geq \operatorname{Per}(B)
$$

where $B$ is an Euclidean ball in $\mathbb{R}^{d}$ with $\mu(B)=\mu(S)$.

In this paper, we consider a discrete analogue of Theorem 5. To state it, we need some more notation. Let $\mathbb{L}^{d}$ denote the graph of the $d$-dimensional integer lattice, i.e. the graph with vertex-set $\mathbb{Z}^{d}$ and edge-set

$$
\left\{\left\{x, x+e_{i}\right\}: x \in \mathbb{Z}^{d}, i \in[d]\right\}
$$

where $e_{i}=(0,0, \ldots, 0,1,0, \ldots, 0)$ denotes the $i$ th unit vector in $\mathbb{R}^{d}$. If $S \subset \mathbb{Z}^{d}$, we let $\partial S$ denote the edge-boundary of $S$ in the graph $\mathbb{L}^{d}$, meaning the set of edges of $\mathbb{L}^{d}$ which join a vertex in $S$ to a vertex not in $S$.

The following edge-isoperimetric inequality in $\mathbb{L}^{d}$ is an easy consequence of the Loomis-Whitney Inequality, and the inequality of arithmetic and geometric means (the AM-GM inequality, for short). It is also an immediate consequence of Theorem 8 in [6].

Theorem 6. Let $S \subset \mathbb{Z}^{d}$ with $|S|<\infty$. Then

$$
|\partial S| \geq 2 d|S|^{(d-1) / d}
$$

Equality holds in Theorem 6 if $S=[a]^{d}$ for some $a \in \mathbb{N}$. Very slightly more generally, equality holds if $S=S_{1} \times S_{2} \times \ldots \times S_{d}$, where $S_{1}, \ldots, S_{d}$ are equal-sized intervals in $\mathbb{Z}$; we will call such a set a cube.

\footnotetext{
${ }^{1}$ The first complete proof, by placing the calculus of variations on a fully rigorous footing, was given by Weierstrass in a series of lectures in the 1870 s in Berlin.
} 


\section{Isoperimetric stability}

In their seminal work [21], Fusco, Maggi and Pratelli proved that if $S \subset \mathbb{R}^{d}$ is a Borel set of finite measure and with distributional perimeter close to the minimum possible size (viz, the size given by Theorem 5), then $S$ must be close in symmetric difference to an Euclidean ball of the same measure, confirming a conjecture of Hall.

Theorem 7 (Fusco, Maggi, Pratelli). Suppose $S \subset \mathbb{R}^{d}$ is a Borel set with Lebesgue measure $\mu(S)<\infty$, and with

$$
\operatorname{Per}(S) \leq(1+\varepsilon) \operatorname{Per}(B)
$$

where $B$ is a Euclidean ball with $\mu(B)=\mu(S)$. Then there exists $x \in \mathbb{R}^{d}$ such that

$$
\mu(S \Delta(B+x)) \leq C_{d} \sqrt{\varepsilon} \mu(S),
$$

where $C_{d}>0$ is a constant depending upond alone.

As observed in [21], Theorem 7 is sharp up to the value of the constant $C_{d}$, as can be seen by taking $S$ to be an ellipsoid with $d-1$ semi-axes of length 1 and one semi-axis of length slightly larger than 1 .

In this paper, we prove a discrete analogue of Theorem 7 by using Theorem 1 . We prove the following stability result for the edge-isoperimetric inequality in $\mathbb{L}^{d}$.

Theorem 8. Let $d \in \mathbb{N}$ with $d \geq 2$. If $S \subset \mathbb{Z}^{d}$ with $|S|<\infty$ and with

$$
|\partial S| \leq(1+\varepsilon) 2 d|S|^{(d-1) / d},
$$

then there exists a cube $C \subset \mathbb{Z}^{d}$ such that

$$
|S \Delta C| \leq 72 d^{5 / 2} \sqrt{\varepsilon}|S| .
$$

Theorem 8 has the best possible dependence on $\varepsilon$, as can be seen by taking a 'cuboid' $S=[a]^{d-1} \times[b]$, where $b$ is slightly larger than $a$ (see Remark 19 for details). We conjecture that the dependence on $d$ could be improved, to $\Theta(\sqrt{d})$ (see section 5).

\subsection{Structure of paper}

In section 2, we cover some background and introduce some notation. In subsection 3.1, we present our main lemmas and prove that they imply our main theorem. In subsection 3.2, we prove the main lemmas. In section 4, we prove Theorem 8, our stability result for the edge-isoperimetric inequality in the lattice $\mathbb{L}^{d}$. Finally, in section 5, we conclude with some open problems.

\section{Preliminaries}

In this section, we state some definitions and known results from probability theory and information theory, and we describe some of our notation. For background concerning the information-theoretic results, and for proofs, the reader is referred to [11].

Throughout this paper, $\log$ means $\log _{2}$, and we use the convention $0 \log 0=0$. 


\section{DAvid Ellis, Ehud Friedgut, Guy Kindler And Amir YehudAyofF}

Definition. Let $p$ be a probability distribution on a finite or countable set $X$. The entropy of $p$ is defined by

$$
H(p):=\sum_{x \in X} p(x) \log (1 / p(x)) .
$$

The entropy of a random variable is the entropy of its distribution. (By a slight abuse of terminology, we will often identify a random variable with its distribution.)

Intuitively, the entropy of a random variable measures the 'amount of information' one has from knowing the value of the random variable.

Let $\operatorname{supp}(p)$ denote the support of the distribution $p$, i.e. $\operatorname{supp}(p)=\{x: p(x) \neq 0\}$. The convexity of $t \mapsto \log (1 / t)$ implies that

$$
H(p) \leq \log |\operatorname{supp}(p)| .
$$

Note that equality holds in (6) iff $p$ is uniformly distributed on its support.

Definition. For two random variables $A, B$ taking values in a set $X$, the conditional entropy of $A$ given $B$ is defined by

$$
H(A \mid B):=H(A, B)-H(B) .
$$

The chain rule for entropy follows immediately:

$$
H(A, B)=H(B)+H(A \mid B) .
$$

It is easy to prove that conditioning does not increase entropy: for any two random variables $A, B$,

$$
H(A \mid B) \leq H(A) .
$$

For three random variables $A, B, C$, we denote by $p(a, b, c)$ the probability of the event $\{A=a, B=$ $b, C=c\}$, we denote by $p(a)$ the probability of the event $\{A=a\}$, and if $p(b)>0$, we denote by $p(a \mid b)$ the probability of $\{A=a\}$ given $\{B=b\}$.

Definition. If $p$ and $q$ are two distributions on a finite or countable set $X$, with $\operatorname{supp}(p) \subset \operatorname{supp}(q)$, the Kullback-Leibler divergence between $p, q$ is defined by

$$
D(p \| q):=\sum_{x} p(x) \log \frac{p(x)}{q(x)} .
$$

If $\operatorname{supp}(p) \not \subset \operatorname{supp}(q)$, we define $D(p \| q):=\infty$.

The Kullback-Leibler divergence is non-negative, it is zero if and only if $p=q$, but it is not symmetric, i.e. in general, $D(q \| p) \neq D(p \| q)$, even when $\operatorname{supp}(p)=\operatorname{supp}(q)$.

Definition. The mutual information of $A$ and $B$ is defined by

$$
I(A ; B)=H(A)-H(A \mid B)=\sum_{a, b} p(a, b) \log \frac{p(a, b)}{p(a) p(b)} .
$$




\section{GeOMETRIC StABILITy VIA InFORMATION THEORY}

Note that this is also the Kullback-Leibler divergence between the joint distribution of $A$ and $B$ and the product of the marginals.

The mutual information of $A$ and $B$, conditioned on $C$, is defined by

$$
I(A ; B \mid C)=H(A \mid C)-H(A \mid(B, C))=\sum_{a, b, c} p(a, b, c) \log \frac{p(a, b \mid c)}{p(a \mid c) p(b \mid c)} .
$$

Mutual information is symmetric under interchanging $A$ and $B$, i.e.

$$
I(B ; A \mid C)=I(A ; B \mid C) .
$$

Another (intuitively plausible) property of mutual information is that if $C$ is a function of $B$, then

$$
I(A ; C) \leq I(A ; B) .
$$

We refer to this property as the 'monotonicity of mutual information'.

Marginal distributions. Let $p$ be a probability distribution on $\mathbb{Z}^{d}$. For a subset $g \subset[d]$, we denote by $p_{g}$ the marginal distribution of $p$ on the set of coordinates $g$, i.e.

$$
\forall S \subset \mathbb{Z}^{g}, \quad p_{g}(S)=p\left(S \times \mathbb{Z}^{[d] \backslash g}\right) .
$$

If $\left(g_{1}, g_{2}, \ldots, g_{r}\right)$ is a partition of $[d]$, we denote by $p_{g_{1}} p_{g_{2}} \ldots p_{g_{r}}$ the obvious product-distribution on $\mathbb{Z}^{d}$.

We will need the following equation relating the divergences between various products of marginals of $p$.

$$
D\left(p \| \prod_{i=1}^{d} p_{i}\right)=\sum_{i=2}^{d} D\left(p_{[i]} \| p_{[i-1]} p_{i}\right) .
$$

This is easily verified, using the definition of $D$ and expanding the logarithms on the right-hand side. Note that if $X=\left(X_{1}, X_{2}, \ldots, X_{d}\right)$ is a random variable with probability distribution $p$, then the left-hand side is precisely the total correlation of the set of random variables $\left\{X_{1}, \ldots, X_{d}\right\}$.

\section{Proof of Theorem 1}

\subsection{Main lemmas and the deduction of Theorem 1}

In this subsection, we present several statements that, when put together, easily imply our main theorem.

First, we would like to show that if the Uniform Cover inequality is close to being tight for a set $S$, then there is not much mutual information between any 1-dimensional marginal of the uniform distribution on $S$, and the complementary $(d-1)$-dimensional marginal.

Lemma 9. Let $d \in \mathbb{N}$ with $d \geq 2$. Let $\mathcal{G} \subset \mathcal{P}([d])$ with $m(\mathcal{G}), \sigma(\mathcal{G})>0$. Let $0 \leq \varepsilon \leq \frac{1}{2}$. Let $S \subset \mathbb{Z}^{d}$ with $|S|<\infty$ and with

$$
|S| \geq(1-\varepsilon)\left(\prod_{g \in \mathcal{G}}\left|\pi_{g}(S)\right|\right)^{1 / m(\mathcal{G})} .
$$


Let $p$ denote the uniform distribution on $S$. Then for all $i \in[d]$, we have

$$
I\left(p_{\{i\}} ; p_{[d] \backslash\{i\}}\right) \leq 2 \rho(\mathcal{G}) \varepsilon
$$

Note that, by monotonicity of mutual information, Lemma 9 implies that for any $J \subset[d]$ and any $i \notin J$, we have

$$
D\left(p_{J \cup\{i\}} \| p_{J} p_{\{i\}}\right)=I\left(p_{\{i\}} ; p_{J}\right) \leq I\left(p_{\{i\}} ; p_{[d] \backslash\{i\}}\right) \leq 2 \rho(\mathcal{G}) \varepsilon .
$$

Given a set $S \subset \mathbb{Z}^{d}$, we want to measure how far the uniform distribution $p$ on $S$ is from the product of some of its marginals. It turns out that a useful measure for us (which we call the 'hole-weight') is the sum of the product of the marginals over all points not in $S$ ('holes'). Formally, if $\left(g_{1}, \ldots, g_{r}\right)$ is a partition of $[d]$, we define the hole-weight of $S$ with respect to $\left(g_{1}, \ldots, g_{r}\right)$ by

$$
\operatorname{Hole}_{g_{1}, \ldots, g_{r}}(S):=\sum_{x \notin S} \prod_{j=1}^{r} p_{g_{j}}(x) \text {. }
$$

In all but one case below, the partition of $[d]$ which defines the hole-weight will be of the form $(\{i\},[d] \backslash$ $\{i\}$ ), so for brevity, we write $\operatorname{Hole}_{i}(S):=\operatorname{Hole}_{\{i\},[d] \backslash\{i\}}(S)$. Also, when stating and proving lemmas, instead of considering $S \subseteq \mathbb{Z}^{\{i\}} \times \mathbb{Z}^{[d] \backslash\{i\}}$, we will sometimes consider the general 'two-dimensional' setting $S \subset X_{1} \times X_{2}$, i.e. $S$ is simply a subset of a product of two sets. If $X_{1}$ and $X_{2}$ are sets, and $S \subset X_{1} \times X_{2}$, we will write $\operatorname{Hole}(S):=\operatorname{Hole}_{\{1\},\{2\}}(S)$.

The following claim bounds from above the hole-weight of $S$ by the Kullback-Leibler divergence between the uniform distribution on $S$, and the product of its marginals.

Claim 10. Let $S \subset \mathbb{Z}^{d}$ with $|S|<\infty$, let $p$ be the uniform distribution on $S$, and let $\left(g_{1}, \ldots g_{r}\right)$ be a partition of $[d]$. Let $p$ be the uniform distribution on $S$, and let $\left(p_{g_{j}}\right)$ denote the corresponding marginals. Then

$$
\text { Hole }_{g_{1}, \ldots g_{r}}(S) \leq D\left(p \| \prod_{j=1}^{r} p_{g_{j}}\right) .
$$

Remark 11. Of course, another very natural way of measuring how far $p$ is from the product of its marginals is to simply use the $\ell^{1}$-distance

$$
\left\|p-\prod_{j=1}^{r} p_{g_{j}}\right\|_{1} .
$$

(Recall that if $p$ and $q$ are probability distributions on a finite or countable set $X$, then the $\ell^{1}$-distance between $p$ and $q$ is defined by

$$
\|p-q\|_{1}:=\sum_{x \in X}|p(x)-q(x)|=2 \max \{p(S)-q(S): S \subset X\} ;
$$

the quantity $\max \{p(S)-q(S): S \subset X\}=\frac{1}{2}\|p-q\|_{1}$ is often called the total variation distance between $p$ and $q$.) 


\section{GEOMETRIC STABILITY VIA INFORMATION THEORY}

One can bound the $\ell^{1}$-distance (12) in terms of the divergence on the right-hand side of (11), using Pinsker's inequality. Pinsker's inequality states that if $p$ and $q$ are two probability distributions on a finite or countable set $X$, then

$$
\|p-q\|_{1} \leq \sqrt{(2 \ln 2) D(p \| q)}
$$

(Note that Pinkser originally proved (13) with a worse constant. The above form, in which the constant is sharp, is due independently to Kullback, Csiszár and Kemperman.) Applying this yields

$$
\left\|p-\prod_{j=1}^{r} p_{g_{j}}\right\|_{1} \leq \sqrt{(2 \ln 2) D\left(p \| \prod_{j=1}^{r} p_{g_{j}}\right)}
$$

Unfortunately, this application of Pinsker's inequality introduces $\Theta(\sqrt{\varepsilon})$-dependence in the conclusion of Theorem 1 (this was our original approach). We obtain $\Theta(\varepsilon)$-dependence by relying only on the hole-weight.

We now need a lemma saying that if the hole-weight of a two-dimensional set $S$ is small, then $S$ is close to a 2-dimensional box.

Lemma 12. Let $X_{1}$ and $X_{2}$ be sets. Let $S \subset X_{1} \times X_{2}$ with $|S|<\infty$. Let $p$ denote the uniform distribution on $S$, and let $p_{1}, p_{2}$ denote its marginals. Let $0<\alpha<1$. Then there exists $R_{1} \subset X_{1}$ such that

$$
p_{1}\left(X_{1} \backslash R_{1}\right) \leq \frac{2 \operatorname{Hole}(S)}{\alpha},
$$

and such that for every $x_{1} \in R_{1}$, we have

$$
p_{1}\left(x_{1}\right) \geq\left(1-\frac{2 \operatorname{Hole}(S)}{\alpha}\right) \cdot \frac{(1-\alpha)}{\left|R_{1}\right|} .
$$

The idea behind Lemma 12 is that the set $R_{1} \subset X_{1}$ is a good candidate to be one of the multiplicands ('edges') of a box approximating $S$ : on the one hand, it captures most of $p_{1}(S)$, and on the other hand, $p_{1}$ restricted to $R_{1}$ is close (in a sense) to being uniform. Indeed, the main step in the proof of Theorem 1 below is to apply Lemma 12, with an appropriate value of $\alpha$, yielding (for each $i \in[d]$ ) a set $R_{i} \subset \mathbb{Z}^{\{i\}}$, and then to show that $S$ is close in symmetric difference to the Cartesian product of the $R_{i}$ 's.

The above lemmas now yield the proof of our main theorem.

Proof of Theorem 1. Let $\mathcal{G} \subset \mathcal{P}([d])$ with $m(\mathcal{G}), \sigma(\mathcal{G})>0$. We may and shall assume that $\varepsilon<\left(\left(4 d^{2}+\right.\right.$ $64 d) \rho(\mathcal{G}))^{-1}$, as otherwise the conclusion of the theorem holds trivially. Given a set $S \subset \mathbb{Z}^{d}$ with $|S|<\infty$, and with

$$
|S| \geq(1-\varepsilon)\left(\prod_{g \in \mathcal{G}}\left|\pi_{g}(S)\right|\right)^{1 / m(\mathcal{G})},
$$

we apply Lemma 9 to deduce that for all $i \in[d]$,

$$
I\left(p_{\{i\}} ; p_{[d] \backslash\{i\}}\right) \leq 2 \rho(\mathcal{G}) \varepsilon .
$$


This implies, via (10) and Claim 10, that for every $i$ in $[d]$,

$$
\operatorname{Hole}_{i}(S) \leq 2 \rho(\mathcal{G}) \varepsilon \text {. }
$$

Next, for each $i \in[d]$, we apply Lemma 12 to $S \subseteq \mathbb{Z}^{\{i\}} \times \mathbb{Z}^{[d] \backslash\{i\}}$ (i.e., we take $X_{1}=\mathbb{Z}^{\{i\}}$ and $X_{2}=\mathbb{Z}^{[d] \backslash\{i\}}$ ), so that $\operatorname{Hole}(S)=\operatorname{Hole}_{i}(S) \leq 2 \rho(\mathcal{G}) \varepsilon$; we take $\alpha=1 / d$. This yields (for every $i \in[d]$ ) a set $R_{i} \subset \mathbb{Z}^{\{i\}}$ such that

$$
p_{i}\left(\mathbb{Z}^{\{i\}} \backslash R_{i}\right) \leq 2 d \cdot \operatorname{Hole}_{i}(S) \leq 4 d \rho(\mathcal{G}) \varepsilon,
$$

and such that for any $x_{i} \in R_{i}$,

$$
p_{i}\left(x_{i}\right) \geq\left(1-2 d \cdot \operatorname{Hole}_{i}(S)\right)(1-1 / d) \frac{1}{\left|R_{i}\right|} \geq(1-4 d \rho(\mathcal{G}) \varepsilon)(1-1 / d) \frac{1}{\left|R_{i}\right|} \geq(1-1 / d)^{2} \frac{1}{\left|R_{i}\right|} .
$$

Let

$$
R:=R_{1} \times R_{2} \times \ldots \times R_{d}
$$

By the union bound,

$$
p(S \backslash R) \leq 4 d^{2} \rho(\mathcal{G}) \varepsilon
$$

i.e.

$$
|S \backslash R| \leq 4 d^{2} \rho(\mathcal{G}) \varepsilon|S| .
$$

On the other hand, for every $x \in R \backslash S$, we have

$$
\prod_{i=1}^{d} p_{i}\left(x_{i}\right) \geq \prod_{i=1}^{d} \frac{(1-1 / d)^{2}}{\left|R_{i}\right|}=\frac{(1-1 / d)^{2 d}}{|R|} \geq \frac{1}{16|R|} .
$$

Hence,

$$
\frac{|R \backslash S|}{16|R|} \leq \sum_{x \in R \backslash S} \prod_{i=1}^{d} p_{i}\left(x_{i}\right) \leq \operatorname{Hole}_{(1,2, \ldots, d)}(S) .
$$

Applying Claim 10 and equation (9), it follows that

$$
\frac{|R \backslash S|}{16|R|} \leq D\left(p \| \prod_{i=1}^{d} p_{i}\right)=\sum_{i=2}^{d} D\left(p_{[i]} \| p_{[i-1]} p_{i}\right) .
$$

Applying the bound (10) gives

$$
\frac{|R \backslash S|}{16|R|} \leq 2 d \rho(\mathcal{G}) \varepsilon
$$

which implies that $|R \backslash S| \leq 32 d \rho(\mathcal{G}) \mathcal{\varepsilon}|R|$. Hence, $|S| \geq(1-32 d \rho(\mathcal{G}) \varepsilon)|R|$, and so

$$
|R \backslash S| \leq \frac{32 d \rho(\mathcal{G}) \varepsilon}{1-32 d \rho(\mathcal{G}) \varepsilon}|S| \leq 64 d \rho(\mathcal{G}) \varepsilon|S| .
$$

Combining (14) and (15) gives

$$
|S \triangle R| \leq\left(4 d^{2}+64 d\right) \rho(\mathcal{G}) \varepsilon|S|,
$$

so we may take $B=R$, completing the proof. 


\section{GEOMETRIC STABILITY VIA INFORMATION THEORY}

For completeness, we now present the deduction of Corollary 2 from Theorem 1, using the approximation argument outlined in [22].

Proof of Corollary 2. Let $m \in \mathbb{N}$. Let $\mathcal{G} \subset \mathcal{P}([d])$ be a uniform $m$-cover. Let $S \subset \mathbb{R}^{d}$ be a body such that

$$
\mu(S) \geq(1-\varepsilon)\left(\prod_{g \in \mathcal{G}} \mu\left(\pi_{g}(S)\right)\right)^{1 / m} .
$$

Fix $\eta \in(0,1)$. Since $\bar{S}$ is compact and $\mu(S)>0$, there exists a compact set $K \subset S$ such that $\mu(K) \geq(1-\eta) \mu(S)$. Choose an open cover $\mathcal{C}$ of $K$ by open cubes with corners at rational coordinates, such that all the cubes in $\mathcal{C}$ are contained within $S$. Since $K$ is compact, we may choose a finite subset $\mathcal{C}^{\prime} \subset \mathcal{C}$ such that $\mathcal{C}^{\prime}$ is a cover of $K$. Choose an axis-parallel grid of some side-length $\delta>0$, which is a common refinement of all the sets in $\mathcal{C}^{\prime}$ (meaning that all the open cubes in $\mathcal{C}^{\prime}$ are unions of open grid-cubes). Let $F$ be the union of all the open grid-cubes which are contained in $S$. Then $\mu(F) \geq(1-\eta) \mu(S)$. Let $N$ be the number of open grid-cubes in $F$, and for each $g \subset[d]$, let $N_{g}$ be the number of (lower-dimensional) open grid-cubes in the projection of $F$ onto the plane $\left\{x: x_{i}=0 \forall i \notin g\right\}$. Then we have

$$
\begin{aligned}
N^{m} \delta^{m d} & =\mu(F)^{m} \\
& \geq(1-\eta)^{m} \mu(S)^{m} \\
& \geq(1-\eta)^{m}(1-\varepsilon)^{m} \prod_{g \in \mathcal{G}} \mu\left(\pi_{g}(S)\right) \\
& \geq(1-\eta)^{m}(1-\varepsilon)^{m} \prod_{g \in \mathcal{G}} N_{g} \delta^{|g|} \\
& =(1-\varepsilon-\eta+\varepsilon \eta)^{m} \delta^{m d} \prod_{g \in \mathcal{G}} N_{g} .
\end{aligned}
$$

Hence, cancelling the common factor of $\delta^{m d}$ and rearranging, we obtain

$$
N \geq(1-\varepsilon-\eta+\varepsilon \eta)\left(\prod_{g \in \mathcal{G}} N_{g}\right)^{1 / m} .
$$

Therefore, by Theorem 1, there exists a box $B_{\eta} \subset \mathbb{R}^{d}$ (which is a union of open grid-cubes), such that

$$
\mu\left(F \Delta B_{\eta}\right) \leq b \rho(\mathcal{G})(\varepsilon+\eta-\varepsilon \eta) \mu(F)
$$

Hence,

$$
\mu\left(S \Delta B_{\eta}\right) \leq b \rho(\mathcal{G})(\varepsilon+\eta-\varepsilon \eta) \mu(S)+\eta \mu(S) .
$$

Since $\eta \in(0,1)$ was arbitrary, it follows by a compactness argument that there exists a box $B \subset \mathbb{R}^{d}$ such that

$$
\mu(S \Delta B) \leq b \rho(\mathcal{G}) \varepsilon \mu(S)
$$




\section{DAvid Ellis, Ehud Friedgut, Guy Kindler And Amir YehudAyofF}

\subsection{Proofs of the main lemmas}

We now present the proofs of our main lemmas.

Proof of Lemma 9. Our proof is information-theoretic, inspired by the technique of Radhakrishnan from [24].

Let $d, \mathcal{G}, \varepsilon, S$ and $p$ be as in the statement of the lemma. Let $X$ be a random variable uniformly distributed on $S$. Assume without loss of generality that $i=d$. Recall that $H(X)=\log (|S|)$ and that by (6), for all $g \subset[d]$, we have $H\left(X_{g}\right) \leq \log \left(\left|\pi_{g}(S)\right|\right)$. Set $m:=m(\mathcal{G}), \sigma:=\sigma(\mathcal{G})$ and $\rho:=\rho(\mathcal{G})$. Since $|S| \geq(1-\varepsilon)\left(\prod_{g \in \mathcal{G}}\left|\pi_{g}(S)\right|\right)^{1 / m}$, we have

$$
\begin{aligned}
H(X) & =\log |S| \geq \log (1-\varepsilon)+\frac{1}{m} \sum_{g \in \mathcal{G}} \log \left|\pi_{g}(S)\right| \geq \log (1-\varepsilon)+\frac{1}{m} \sum_{g \in \mathcal{G}} H\left(X_{g}\right) \\
& \geq-2 \varepsilon+\frac{1}{m} \sum_{g \in \mathcal{G}} H\left(X_{g}\right) .
\end{aligned}
$$

Hence,

$$
\begin{aligned}
& 2 \varepsilon \geq \frac{1}{m} \sum_{g \in \mathcal{G}} H\left(X_{g}\right)-H(X) \\
& =\frac{1}{m} \sum_{g \in \mathcal{G}} H\left(X_{g}\right)-H\left(X_{d}\right)-H\left(X_{[d-1]} \mid X_{d}\right) \quad \text { (by the chain rule) } \\
& =\frac{\sum_{\substack{g \in \mathcal{G} \\
d \in g}}\left(H\left(X_{d}\right)+H\left(X_{g \backslash\{d\}} \mid X_{d}\right)\right)+\sum_{\substack{g \in \mathcal{G} \\
d \notin g}} H\left(X_{g}\right)}{m}-H\left(X_{d}\right)-H\left(X_{[d-1]} \mid X_{d}\right) \\
& \geq \frac{\sum_{\substack{g \in \mathcal{G} \\
d \in g}} H\left(X_{g \backslash\{d\}} \mid X_{d}\right)+\sum_{\substack{g \in \mathcal{G} \\
d \notin g}} H\left(X_{g}\right)}{m}-H\left(X_{[d-1]} \mid X_{d}\right) \\
& \text { (since, by definition of } m=m(\mathcal{G}) \text {, there are at least } m \text { sets } g \in \mathcal{G} \text { with } d \in g \text { ) } \\
& =\sum_{j<d}\left(\frac{\sum_{\substack{g \in \mathcal{G} \\
d, j \in g}} H\left(X_{j} \mid X_{g \cap[j-1]}, X_{d}\right)+\sum_{\substack{g \in \mathcal{G}: \\
g \cap\{d, j\}=j}} H\left(X_{j} \mid X_{g \cap[j-1]}\right)}{m}-H\left(X_{j} \mid X_{[j-1]}, X_{d}\right)\right) \\
& \geq \sum_{j<d}\left(\frac{\sum_{\substack{g \in \mathcal{G}: \\
d, j \in g}} H\left(X_{j} \mid X_{[j-1]}, X_{d}\right)+\sum_{\substack{g \in \mathcal{G}: \\
g \cap\{, j\}=j}} H\left(X_{j} \mid X_{[j-1]}\right)}{m}-H\left(X_{j} \mid X_{[j-1]}, X_{d}\right)\right) \\
& \text { (since extra conditioning does not increase entropy) } \\
& \geq \frac{\sigma}{m} \sum_{j<d}\left(H\left(X_{j} \mid X_{[j-1]}\right)-H\left(X_{j} \mid X_{[j-1]}, X_{d}\right)\right) \quad \text { (using the definitions of } m \text { and } \sigma \text { ) } \\
& =\rho^{-1}\left(H\left(X_{[d-1]}\right)-H\left(X_{[d-1]} \mid X_{d}\right)\right) \quad \text { (by the chain rule) } \\
& =\rho^{-1} I\left(X_{[d-1]} ; X_{d}\right) \text {, }
\end{aligned}
$$

as required. 


\section{GEOMETRIC STABILITY VIA INFORMATION THEORY}

Remark 13. As mentioned in the Introduction, Lemma 9 can also be proved by appealing to an entropy inequality in the paper [1] of Balister and Bollobás. Indeed, without loss of generality taking $i=d$, it suffices to prove the inequality

$$
\sigma H\left(X_{d}\right)+\sigma H\left(X_{[d-1]}\right)+(m-\sigma) H(X) \leq \sum_{g \in \mathcal{G}} H\left(X_{g}\right)
$$

which follows from two applications of Theorem 6 in [1], the first application being with $\mathcal{A}=\{g \in \mathcal{G}$ : $d \notin \mathcal{G}\}$. We have opted to give the self-contained proof above, as it is not much longer.

Proof of Claim 10. Recall that $\log \frac{1}{1-\alpha} \geq \alpha$ for all $\alpha \in[0,1)$, since $1-\alpha \leq e^{-\alpha} \leq 2^{-\alpha}$. Using this, and the convexity of $t \mapsto \log (1 / t)$, we have

$$
\begin{aligned}
D\left(p \| \prod_{j=1}^{r} p_{g_{j}}\right) & =-\sum_{x \in S} p(x) \log \frac{\prod_{j=1}^{r} p_{g_{j}}\left(x_{g_{j}}\right)}{p(x)} \\
& \geq-\log \sum_{x \in S} p(x) \frac{\prod_{j=1}^{r} p_{g_{j}}\left(x_{g_{j}}\right)}{p(x)} \\
& =\log \frac{1}{1-\sum_{x \notin S} \prod_{j=1}^{r} p_{g_{j}}\left(x_{g_{j}}\right)} \\
& \geq \sum_{x \notin S} \prod_{j=1}^{r} p_{g_{j}}\left(x_{g_{j}}\right) \\
& =\operatorname{Hole}_{g_{1}, \ldots g_{r}}(S) .
\end{aligned}
$$

\section{Trimming a 2-dimensional set to yield a rectangle}

In this subsection, we deal with the most combinatorially-flavoured ingredient in our paper, the proof of Lemma 12. Given a set $S \subset X_{1} \times X_{2}$, we proceed to trim away some of its 'vertical fibres' (sets of the form $\left(\left\{x_{1}\right\} \times X_{2}\right) \cap S$, for some $\left.x_{1} \in X_{1}\right)$, in such a way that none of the remaining fibres is very short compared to the others. This leaves us with a subset $R_{1} \subset X_{1}$, such that weight of each remaining vertical fibre of $R_{1}$ is at least a constant fraction of the average weight of the remaining fibres. The total amount of mass trimmed is bounded from above in terms of $\operatorname{Hole}(S)$. Applying this procedure with $\alpha=1 / 2$, and then repeating it to find a similar set $R_{2} \subset X_{2}$, yields the following.

Lemma 14. Let $X_{1}$ and $X_{2}$ be sets. If $S \subset X_{1} \times X_{2}$ with $|S|<\infty$, then there exist $R_{1} \subset X_{1}$ and $R_{2} \subset X_{2}$ such that

$$
\left|S \Delta\left(R_{1} \times R_{2}\right)\right| \leq 20 \text { Hole }(S)|S|
$$

We will not use this lemma directly in our proof of our main theorem, but its proof is included for the reader's interest at the end of this subsection. 
Proof of Lemma 12. For $j \in\{1,2\}$, let $\pi_{j}(S)$ denote the natural projection of $S$ onto $X_{j}$. We find an appropriate set $R_{1}$ by iteratively removing from $\pi_{1}(S)$ those points $x_{1}$ for which $p_{1}\left(x_{1}\right)$ is too 'small', namely, those points whose fibre has size less than $(1-\alpha)$ times the average size of a fibre.

To this end, we define recursively: $T^{(0)}:=\emptyset$ and $R^{(1)}:=\pi_{1}(S)$, and for each $j \in \mathbb{N}$,

$$
\begin{aligned}
T^{(j)} & :=\left\{x_{1} \in R^{(j)}: \frac{p_{1}\left(x_{1}\right)}{p_{1}\left(R^{(j)}\right)} \leq \frac{1-\alpha}{\left|R^{(j)}\right|}\right\}, \\
R^{(j+1)} & :=\pi_{1}(S) \backslash \bigcup_{r=1}^{j} T^{(r)} \\
\varepsilon^{(j)} & :=p_{1}\left(T^{(j)}\right) .
\end{aligned}
$$

Define the limit objects:

$$
\begin{aligned}
U^{(\infty)} & :=\bigcup_{j=1}^{\infty} T^{(j)} \\
R_{1} & :=\pi_{1}(S) \backslash U^{(\infty)}, \\
\varepsilon_{1} & :=p_{1}\left(U^{(\infty)}\right)=\sum_{j=1}^{\infty} \varepsilon^{(j)} .
\end{aligned}
$$

Since $S$ is finite, this process stabilizes after finitely many steps. If we can show that

$$
\varepsilon_{1}=p_{1}\left(\pi_{1}(S) \backslash R_{1}\right) \leq \frac{2 \operatorname{Hole}(S)}{\alpha},
$$

then by definition, for every $x_{1} \in R_{1}$, we indeed have

$$
p_{1}\left(x_{1}\right) \geq p_{1}\left(R_{1}\right) \cdot \frac{1-\alpha}{\left|R_{1}\right|} \geq\left(1-\frac{2 \operatorname{Hole}(S)}{\alpha}\right) \cdot \frac{(1-\alpha)}{\left|R_{1}\right|}
$$

proving Lemma 12.

To obtain (17), it suffices to prove the following claim.

Claim 15. $\alpha \sum_{j=1}^{\infty} \varepsilon^{(j)}\left(1-\sum_{1 \leq r<j} \varepsilon^{(r)}\right) \leq \operatorname{Hole}(S)$.

Indeed, Claim 15 implies

$$
\begin{aligned}
\frac{1}{\alpha} \operatorname{Hole}(S) & \geq \sum_{j=1}^{\infty} \varepsilon^{(j)}\left(1-\sum_{1 \leq r<j} \varepsilon^{(r)}\right) \\
& =\left(\sum_{j=1}^{\infty} \varepsilon^{(j)}\right)-\left(\sum_{j=1}^{\infty} \sum_{1 \leq r<j} \varepsilon^{(j)} \varepsilon^{(r)}\right) \geq \varepsilon_{1}-\frac{1}{2} \varepsilon_{1}^{2} \geq \frac{\varepsilon_{1}}{2}
\end{aligned}
$$

implying (17). 


\section{GEOMETRIC STABILITy VIA INFORMATION THEORY}

It remains, therefore, to prove Claim 15.

Proof of Claim 15. Fix $j \in \mathbb{N}$ and let $x_{1} \in T^{(j)}$. Let

$$
Y=Y\left(x_{1}\right):=\left\{y \in \pi_{2}(S):\left(x_{1}, y\right) \in S\right\}
$$

be the fibre of $x_{1}$, and let $\bar{Y}=\bar{Y}\left(x_{1}\right)=\pi_{2}(S) \backslash Y$. We first bound the mass of the set of elements of $S$ whose projection lies in $Y$.

By definition, we have

$$
p_{1}\left(R^{(j)}\right)=1-\sum_{r<j} \varepsilon^{(r)}
$$

and

$$
p_{1}\left(x_{1}\right) \leq \frac{(1-\alpha) p_{1}\left(R^{(j)}\right)}{\left|R^{(j)}\right|} .
$$

So

$$
\left|R^{(j)} \times Y\right|=\left|R^{(j)}\right||Y|=\left|R^{(j)}\right| \cdot p_{1}\left(x_{1}\right)|S| \leq(1-\alpha)|S|\left(1-\sum_{r<j} \varepsilon^{(r)}\right) .
$$

We therefore have

$$
\begin{aligned}
\sum_{y \in Y} p_{2}(y) & =\sum_{y \in Y} \sum_{x_{1}^{\prime} \in \pi_{1}(S)} p\left(x_{1}^{\prime}, y\right) \\
& \leq \frac{\left|R^{(j)} \times Y\right|}{|S|}+\sum_{y \in Y} \sum_{x_{1}^{\prime} \notin R^{(j)}} p\left(x_{1}^{\prime}, y\right) \\
& \leq(1-\alpha)\left(1-\sum_{r<j} \varepsilon^{(r)}\right)+\sum_{x_{1}^{\prime} \notin R^{(j)}} \sum_{y \in \pi_{2}(S)} p\left(x_{1}^{\prime}, y\right) \\
& =(1-\alpha)\left(1-\sum_{r<j} \varepsilon^{(r)}\right)+\sum_{r<j} \varepsilon^{(r)} \\
& =1-\alpha+\alpha \sum_{r<j} \varepsilon^{(r)} .
\end{aligned}
$$

It follows that

$$
\sum_{y \in \bar{Y}} p_{2}(y) \geq 1-\left(1-\alpha+\alpha \sum_{r<j} \varepsilon^{(r)}\right)=\alpha\left(1-\sum_{r<j} \varepsilon^{(r)}\right)
$$

Now summing over $x_{1}$ and using (18), we have

$$
\sum_{x_{1} \in T^{(j)}} p_{1}\left(x_{1}\right) \sum_{y \in \bar{Y}\left(x_{1}\right)} p_{2}(y) \geq \varepsilon^{(j)} \alpha\left(1-\sum_{r<j} \varepsilon^{(r)}\right) .
$$


Finally, summing over all $j$, and recalling that if $y \in \bar{Y}\left(x_{1}\right)$ then $\left(x_{1}, y\right) \notin S$, we have

$$
\operatorname{Hole}(S)=\sum_{\left(x_{1}, y\right) \notin S} p_{1}\left(x_{1}\right) p_{2}(y) \geq \alpha \sum_{j=1}^{\infty} \varepsilon^{(j)}\left(1-\sum_{r<j} \varepsilon^{(r)}\right)
$$

This completes the proof of Claim 15, and thus of Lemma 12.

Proof of Lemma 14. We may assume that $\operatorname{Hole}(S)<1 / 20$, otherwise the conclusion of the lemma holds with $R_{1}=R_{2}=\emptyset$. Let $R_{1} \subset X_{1}$ be the set given by Lemma 12 with $\alpha=1 / 2$. Let $R_{2} \subset X_{2}$ be the set given by Lemma 12 with $\alpha=1 / 2$, when coordinates 1 and 2 are interchanged. Let $R=R_{1} \times R_{2}$. By the union bound, we have

$$
\frac{|S \backslash R|}{|S|} \leq 2 \cdot \frac{2 \operatorname{Hole}(S)}{\alpha}=8 \operatorname{Hole}(S)
$$

On the other hand, for every $x \in R$, we have

$$
p_{1}\left(x_{1}\right) p_{2}\left(x_{2}\right) \geq\left(1-\frac{2 \operatorname{Hole}(S)}{\alpha}\right)^{2}(1-\alpha)^{2} \frac{1}{|R|}>\frac{1}{7|R|} .
$$

Therefore,

$$
\operatorname{Hole}(S) \geq \sum_{x \in R \backslash S} p_{1}\left(x_{1}\right) p_{2}\left(x_{2}\right) \geq|R \backslash S| \cdot \frac{1}{7|R|},
$$

which implies that $|R \backslash S| \leq 7 \operatorname{Hole}(S)|R|$. It follows that

$$
|S| \geq|S \cap R| \geq(1-7 \operatorname{Hole}(S))|R| \geq 13|R| / 20,
$$

and therefore

$$
|R \backslash S| \leq 12 \operatorname{Hole}(S)|S|
$$

Combining (19) and (20), we have $|S \triangle R| \leq 20$ Hole $(S)|S|$, completing the proof.

\section{A stability result for the edge-isoperimetric inequality in $\mathbb{L}^{d}$}

In this section, we prove Theorem 8, our stability result for the edge-isoperimetric inequality in the lattice $\mathbb{L}^{d}$, using Theorem 1 and some additional combinatorial arguments.

We start with a short proof of the isoperimetric inequality (Theorem 6), as it will be useful to refer to it later.

Proof of Theorem 6. We write $\partial_{i}(S)$ for the set of edges in $\partial S$ of the form $\left\{x, x+e_{i}\right\}$, i.e. the set of all direction- $i$ edges of $\partial S$. Since $|S|<\infty$, for each $x \in S$, there are at least two direction- $i$ edges of $\partial S$ which project to $\pi_{[d] \backslash\{i\}}(x)$, and therefore

$$
\left|\partial_{i}(S)\right| \geq 2\left|\pi_{[d] \backslash\{i\}}(S)\right|
$$




\section{GEOMETRIC STABILITY VIA INFORMATION THEORY}

Summing over all $i$, we obtain

$$
|\partial S|=\sum_{i=1}^{d}\left|\partial_{i}(S)\right| \geq 2 \sum_{i=1}^{d}\left|\pi_{[d] \backslash\{i\}}(S)\right|
$$

The AM-GM inequality and the Loomis-Whitney inequality yield

$$
|\partial S| \geq 2 \sum_{i=1}^{d}\left|\pi_{[d] \backslash\{i\}}(S)\right| \geq 2 d\left(\prod_{i=1}^{d}\left|\pi_{[d] \backslash\{i\}} S\right|\right)^{1 / d} \geq 2 d|S|^{(d-1) / d} .
$$

The following easy stability result for the AM-GM inequality will be useful in our proof of Theorem 8.

Proposition 16. Let $0 \leq \varepsilon \leq 1 /(16 d)$. Let $z_{1} \geq z_{2} \geq \ldots \geq z_{d}>0$ be such that

$$
\frac{1}{d} \sum_{i=1}^{d} z_{i} \leq(1+\varepsilon)\left(\prod_{i=1}^{d} z_{i}\right)^{1 / d}
$$

Let $G:=\left(\prod_{i=1}^{d} z_{i}\right)^{1 / d}$. Then

$$
\forall i \in[d], \quad(1-2 d \sqrt{d \varepsilon}) G \leq z_{i} \leq(1+2 \sqrt{d \varepsilon}) G
$$

Proof. First, we assert that

$$
z_{1} \leq(1+4 \sqrt{d \varepsilon}) G
$$

Let $\eta \geq 0$ be such that $z_{1}=(1+\eta) G$, and let $\eta_{0}:=4 \sqrt{d \varepsilon}$; then $\eta_{0} \leq 1 / 2$. Assume for a contradiction that $\eta>\eta_{0}$. Then

$$
\begin{aligned}
(1+\varepsilon) G d & \geq \sum_{i=1}^{d} z_{i} \\
& \geq z_{1}+(d-1)\left(\prod_{i=2}^{d} z_{i}\right)^{1 /(d-1)} \\
& =z_{1}+(d-1) G^{d /(d-1)} z_{1}^{-1 /(d-1)} \\
& =G\left(1+\eta+(d-1)(1+\eta)^{-1 /(d-1)}\right) .
\end{aligned}
$$

The function $f: \eta \mapsto \eta+(d-1)(1+\eta)^{-1 /(d-1)}$ has $f^{\prime}(\eta)>0$ for all positive $\eta$, and is therefore strictly 
increasing on $[0, \infty)$. Hence,

$$
\begin{aligned}
(1+\varepsilon) d & \geq 1+\eta+(d-1)(1+\eta)^{-1 /(d-1)} \\
& >1+\eta_{0}+(d-1)\left(1+\eta_{0}\right)^{-1 /(d-1)} \\
& \geq d+\frac{d}{2(d-1)} \eta_{0}^{2}-\frac{d(2 d-1)}{6(d-1)^{2}} \eta_{0}^{3} \\
& \geq d+\left(\frac{d}{2(d-1)} \eta_{0}^{2}-\frac{d(2 d-1)}{12(d-1)^{2}}\right) \eta_{0}^{2} \\
& \geq d+\frac{1}{4} \eta_{0}^{2}
\end{aligned}
$$

contradicting the definition of $\eta_{0}$.

It follows from (22) that

$$
G d \leq \sum_{i=1}^{d} z_{i} \leq(d-1)\left(1+\eta_{0}\right) G+z_{d}
$$

so

$$
z_{d} \geq G\left(1-(d-1) \eta_{0}\right) \geq G\left(1-d \eta_{0}\right)
$$

completing the proof.

Proof of Theorem 8. Let $S \subset \mathbb{Z}^{d}$ with $|S|<\infty$ and

$$
|\partial S| \leq 2 d|S|^{(d-1) / d}(1+\varepsilon)
$$

We may assume that

$$
\varepsilon \leq \frac{1}{72^{2} d^{5}}
$$

otherwise the conclusion of the theorem holds trivially with $C=\emptyset$.

By (21) and (23), we have

$$
\left(\prod_{i=1}^{d}\left|\pi_{[d] \backslash\{i\}} S\right|\right)^{1 /(d-1)} \leq\left(\frac{|\partial S|}{2 d}\right)^{d /(d-1)} \leq(1+\varepsilon)^{d /(d-1)}|S| \leq \frac{1}{1-2 \varepsilon}|S| .
$$

Corollary 3 now implies that there exists a box

$$
R=R_{1} \times R_{2} \times \ldots \times R_{d} \subset \mathbb{Z}^{d}
$$

such that

$$
|S \triangle R| \leq 2\left(64 d+4 d^{2}\right)(d-1) \varepsilon|S| \leq 72 d^{3} \varepsilon|S| \leq \delta|S|,
$$

where

$$
\delta:=\sqrt{d \varepsilon} .
$$

Our aim is to show that $R$ is close in symmetric difference to a cube. 
Clearly, by (26), we have

$$
(1-\delta)|S| \leq|R| \leq(1+\delta)|S|
$$

Let

$$
G:=\left(\prod_{i \in[d]}\left|\pi_{[d] \backslash\{i\}} S\right|\right)^{1 / d} \quad \text { and } \quad a:=|S|^{1 / d} .
$$

Note that, by (25) and the Loomis-Whitney inequality, we have

$$
a^{d-1}=|S|^{(d-1) / d} \leq G \leq(1+\varepsilon)|S|^{(d-1) / d}=(1+\varepsilon) a^{d-1} .
$$

By (21) and (23), we have

$$
\frac{1}{d} \sum_{i=1}^{d}\left|\pi_{[d] \backslash\{i\}}(S)\right| \leq \frac{|\partial S|}{2 d} \leq(1+\varepsilon)|S|^{(d-1) / d} \leq(1+\varepsilon)\left(\prod_{i \in[d]}\left|\pi_{[d] \backslash\{i\}}(S)\right|\right)^{1 / d} .
$$

Hence, by Proposition 16, all the $(d-1)$-dimensional projections of $S$ are of roughly equal size:

$$
\forall i \in[d], \quad(1-2 d \delta) G \leq\left|\pi_{[d] \backslash\{i\}}(S)\right| \leq(1+2 \delta) G .
$$

We now show that all the 1-dimensional projections of $R$ are of roughly equal size.

Claim 17. For every $i \in[d]$, we have $(1-7 \delta) a \leq\left|R_{i}\right| \leq(1+14 d \delta) a$.

Proof. Without loss of generality, we prove the claim for $i=1$. Observe that

$$
(1-2 \delta)\left|\pi_{\{2, \ldots, d\}}(R)\right| \leq\left|\pi_{\{2, \ldots, d\}}(S)\right| .
$$

Indeed, if this does not hold, then, using (27), we have

$$
|S \triangle R| \geq|R \backslash S|>2 \delta|R| \geq 2(1-\delta)|S| \geq \delta|S|,
$$

contradicting (26). Therefore, using (27), (30), (29) and (28) successively, we have

$$
\begin{aligned}
\left|R_{1}\right| & =\frac{|R|}{\left|\pi_{\{2,3, \ldots, d\}}(R)\right|} \\
& \geq \frac{(1-2 \delta)(1-\delta)|S|}{\left|\pi_{\{2, \ldots, d\}}(S)\right|} \\
& \geq \frac{(1-2 \delta)(1-\delta)|S|}{(1+2 \delta) G} \\
& \geq \frac{(1-2 \delta)(1-\delta)|S|}{(1+2 \delta)(1+\varepsilon)|S|^{(d-1) / d}} \\
& \geq(1-7 \delta)|S|^{1 / d} \\
& =(1-7 \delta) a .
\end{aligned}
$$


Similarly, we have

$$
\forall i \in[d], \quad\left|R_{i}\right| \geq(1-7 \delta) a .
$$

Hence, using (27) and (31) we obtain

$$
\begin{aligned}
\left|R_{1}\right| & =\frac{|R|}{\prod_{i=2}^{d}\left|R_{j}\right|} \\
& \leq \frac{(1+\delta)|S|}{(1-7 \delta)^{d-1}|S|^{(d-1) / d}} \\
& \leq(1+14 d \delta)|S|^{1 / d} \\
& =(1+14 d \delta) a .
\end{aligned}
$$

Next, for each $i \in[d]$ we throw away the elements $c \in R_{i}$ such that $\left\{x \in R: x_{i}=c\right\}$ contains few elements of $S$, producing a slightly smaller box $R^{\prime}$.

Fix $i \in[d]$; without loss of generality, $i=1$. Define

$$
Q:=R_{2} \times R_{3} \times \ldots \times R_{d} .
$$

For each $c \in R_{1}$, call $c$ heavy if $\left|S \cap\left\{x \in R: x_{1}=c\right\}\right| \geq 7|Q| / 8$, and call $c$ light otherwise. We assert that there are at most $16 \delta\left|R_{1}\right|$ light elements in $R_{1}$. Indeed, if the number of light elements is larger than this, then

$$
|S \triangle R| \geq|R \backslash S|>(|Q| / 8) \cdot 16 \delta\left|R_{1}\right|=2 \delta|R| \geq 2 \delta(1-\delta)|S| \geq \delta|S|,
$$

contradicting (26).

Let $R_{1}^{\prime}:=\left\{c \in R_{1}: c\right.$ is heavy $\}$. Then $\left|R_{1} \backslash R_{1}^{\prime}\right| \leq 16 \delta\left|R_{1}\right|$. Define $R_{i}^{\prime}$ similarly for each $i \in$ $\{2,3, \ldots, d\}$, and let

$$
R^{\prime}=R_{1}^{\prime} \times R_{2}^{\prime} \times \ldots \times R_{d}^{\prime}
$$

By the union bound, we have

$$
\left|R \backslash R^{\prime}\right| \leq 16 d \delta|R|
$$

and therefore

$$
\left|R^{\prime}\right| \geq(1-16 d \delta)|R|
$$

Our next step is to show that, for each $i \in[d], R_{i}^{\prime}$ occupies most of the interval in which it is contained, as if not, $|\partial S|$ would be too large.

Claim 18. For each $i \in[d]$, we have $\max \left(R_{i}^{\prime}\right)-\min \left(R_{i}^{\prime}\right)+1-\left|R_{i}\right| \leq 8 \delta\left|R_{i}\right|$.

Proof. Without loss of generality, we prove the claim for $i=1$. Let

$$
u:=\min \left(R_{1}^{\prime}\right), \quad v:=\max \left(R_{1}^{\prime}\right) .
$$

Suppose for a contradiction that

$$
v-u+1-\left|R_{1}\right|>8 \delta\left|R_{1}\right| .
$$


Define

$$
\begin{aligned}
& U:=\pi_{\{2,3, \ldots, d\}}\left(S \cap\left\{x \in R: x_{1}=u\right\}\right), \\
& V:=\pi_{\{2,3, \ldots, d\}}\left(S \cap\left\{x \in R: x_{1}=v\right\}\right), \\
& F:=\left\{y \in Q:\left(x_{1}, y\right) \in S \forall x_{1} \in\{u, u+1, \ldots, v-1, v\}\right\}
\end{aligned}
$$

note that $U, V, F \subset Q$. Since $|S|<\infty$, for each $z \in U$, there is at least one edge in $\partial_{1}(S)$ of the form $\left\{\left(x_{1}, z\right),\left(x_{1}+1, z\right)\right\}$, where $x_{1}<u$. Similarly, for each $z \in V$, there is at least one edge in $\partial_{1}(S)$ of the form $\left\{\left(x_{1}, z\right),\left(x_{1}+1, z\right)\right\}$, where $x_{1} \geq v$. Moreover, for each $z \in(U \cap V) \backslash F$, there is at least one edge in $\partial_{1}(S)$ of the form $\left\{\left(x_{1}, z\right),\left(x_{1}+1, z\right)\right\}$ where $u \leq x_{1}<v$. It follows that

$$
\begin{aligned}
\left|\partial_{1}(S)\right| & \geq|U|+|V|+|(U \cap V) \backslash F| \\
& =|U|+|V|+|U \cap V|-|F| \\
& \geq \frac{7}{8}|Q|+\frac{7}{8}|Q|+\frac{3}{4}|Q|-|F| \\
& =\frac{5}{2}|Q|-|F| .
\end{aligned}
$$

We now assert that

$$
|F| \leq \frac{1}{4}|Q|
$$

Indeed, suppose on the contrary that (36) does not hold. By (34), the interval $\{u, u+1, \ldots, v\}$ contains more than $8 \delta\left|R_{1}\right|$ elements not in $R_{1}$. Therefore,

$$
|S \backslash R|>8 \delta\left|R_{1}\right| \cdot|F|>8 \delta\left|R_{1}\right| \cdot \frac{1}{4}|Q|=2 \delta|R| \geq \delta|S|,
$$

contradicting (26).

Using Claim 17 and (26), we have

$$
|Q|=\frac{|R|}{\left|R_{1}\right|} \geq \frac{(1-\delta) a^{d}}{(1+14 d \delta) a} \geq(1-15 d \delta) a^{d-1} .
$$

Hence, by (36) and (35), we have

$$
\left|\partial_{1}(S)\right| \geq 2|Q|+\frac{1}{4}|Q| \geq\left(2+\frac{1}{4}\right)(1-15 d \delta) a^{d-1} .
$$

But we also have

$$
\forall i \in\{2,3, \ldots, d\}, \quad\left|\partial_{i}(S)\right| \geq 2\left|\pi_{[d] \backslash\{i\}}(S)\right| \geq 2(1-2 d \delta) G \geq 2(1-2 d \delta) a^{d-1},
$$

using (28) and (29). Hence, using (24), we have

$$
\begin{aligned}
|\partial S| & =\sum_{i=1}^{d}\left|\partial_{i}(S)\right| \\
& \geq\left(2+\frac{1}{4}\right)(1-15 d \delta) a^{d-1}+(d-1) \cdot 2(1-2 d \delta) a^{d-1} \\
& \geq 2 d a^{d-1}\left(1+\frac{1}{16 d}-2 d \delta\right) \\
& >2 d a^{d-1}(1+\varepsilon),
\end{aligned}
$$

contradicting (23). 
We can now complete the proof of the theorem. Let

$$
L=\max \left\{\max \left(R_{i}^{\prime}\right)-\min \left(R_{i}^{\prime}\right)+1: i \in[d]\right\} .
$$

Assume without loss of generality that

$$
L=\max \left(R_{1}^{\prime}\right)-\min \left(R_{1}^{\prime}\right)+1 .
$$

Define

$$
C=\prod_{i=1}^{d}\left\{\min \left(R_{i}^{\prime}\right), \min \left(R_{i}^{\prime}\right)+1, \ldots, \min \left(R_{i}^{\prime}\right)+L-1\right\} .
$$

The set $C$ is a cube of side-length $L$ containing $R^{\prime}$. Using Claims 17 and 18, we have

$$
\begin{aligned}
L-a & =\max \left(R_{1}^{\prime}\right)-\min \left(R_{1}^{\prime}\right)+1-\left|R_{1}\right|+\left(\left|R_{1}\right|-a\right) \\
& \leq 8 \delta(1+14 d \delta) a+14 d \delta a \\
& \leq 20 d \delta a .
\end{aligned}
$$

Therefore, using (33) and (27), we have

$$
\begin{aligned}
\left|R^{\prime} \triangle C\right| & =L^{d}-\left|R^{\prime}\right| \\
& \leq(1+20 d \delta)^{d} a^{d}-(1-16 d \delta)(1-\delta) a^{d} \\
& \leq\left(1+40 d^{2} \delta\right) a^{d}-(1-17 d \delta) a^{d} \\
& \leq 50 d^{2} \delta a^{d} .
\end{aligned}
$$

Finally, using (32), (26) and (27), we obtain

$$
\begin{aligned}
|S \triangle C| & \leq|S \triangle R|+\left|R \triangle R^{\prime}\right|+\left|R^{\prime} \triangle C\right| \\
& \leq \delta a^{d}+16 d \delta(1+\delta) a^{d}+50 d^{2} \delta a^{d} \\
& \leq 60 d^{2} \delta a^{d} \\
& =60 d^{5 / 2} \sqrt{\varepsilon}|S|,
\end{aligned}
$$

proving the theorem.

Remark 19. As observed in the Section 1, Theorem 8 is sharp up to a constant factor depending upon $d$ alone. To see this, take $S=[a]^{d-1} \times[b]$, where $b=(1+\phi) a, \phi>0$ and $a, b \in \mathbb{N}$, i.e. $S$ is a 'cuboid'. Then

$$
|S|=a^{d-1} b=(1+\phi) a^{d}
$$

and

$$
|S \triangle C| \geq(b-a) a^{d-1}=\phi a^{d}=\frac{\phi}{1+\phi}|S|
$$


for all cubes $C \subset \mathbb{Z}^{d}$. On the other hand, we have

$$
\begin{aligned}
|\partial S|-2 d|S|^{(d-1) / d} & =2 a^{d-1}+2(d-1) a^{d-2} b-2 d\left(a^{d-1} b\right)^{(d-1) / d} \\
& =2 a^{d-1}\left(1+(d-1)(1+\phi)-d(1+\phi)^{(d-1) / d}\right) \\
& \leq 2 a^{d-1}\left(d+\phi(d-1)-d\left[1+\frac{d-1}{d} \phi-\frac{d-1}{2 d^{2}} \phi^{2}\right]\right)=\frac{d-1}{d} \phi^{2} a^{d-1} .
\end{aligned}
$$

Hence, we have $|\partial S|=(1+\varepsilon) 2 d|S|^{(d-1) / d}$, where

$$
\varepsilon=\frac{|\partial S|-2 d|S|^{(d-1) / d}}{2 d|S|^{(d-1) / d}} \leq \frac{\frac{d-1}{d} \phi^{2} a^{d-1}}{2 d a^{d-1}(1+\phi)^{(d-1) / d}} \leq \frac{\phi^{2}}{2 d},
$$

but $S$ is $\delta$-far from any cube, where

$$
\delta=\frac{\phi}{1+\phi} \geq \frac{1}{2} \phi \geq \frac{1}{2} \sqrt{2 d \varepsilon}
$$

\section{Conclusion and open problems}

We have proved stability results for the Uniform Cover inequality (Theorem 1), and the edge-isoperimetric inequality in the $d$-dimensional integer lattice (Theorem 8).

We conjecture that the dependence on $d$ in Theorem 1 can be removed:

Conjecture 20. Let $d \in \mathbb{N}$ with $d \geq 2$. Let $S \subset \mathbb{Z}^{d}$ with $|S|<\infty$. If

$$
|S| \geq(1-\varepsilon)\left(\prod_{g \in \mathcal{G}}\left|\pi_{g} S\right|\right)^{1 / m(\mathcal{G})}
$$

then there exists a box $B \subset \mathbb{Z}^{d}$ such that

$$
|S \Delta B| \leq c \rho(\mathcal{G}) \varepsilon|S|
$$

where $c$ is an absolute constant.

We also conjecture that the dependence upon $d$ in Theorem 8 can be improved to $\Theta(\sqrt{d})$ :

Conjecture 21. Let $S \subset \mathbb{Z}^{d}$ with $|S|<\infty$ and with

$$
|\partial S| \leq 2 d|S|^{(d-1) / d}(1+\varepsilon)
$$

Then there exists a cube $C \subset \mathbb{Z}^{d}$ such that $|S \Delta C| \leq c^{\prime} \sqrt{d \varepsilon}|S|$, where $c^{\prime}$ is an absolute constant.

This would be sharp up to the value of $c^{\prime}$, by the cuboid example in Remark 19.

\section{Acknowledgments}

The authors would like to thank Itai Benjamini and Emanuel Milman for helpful conversations and comments, and an anonymous referee for several helpful suggestions and remarks. 


\section{DAvid Ellis, Ehud Friedgut, Guy Kindler And Amir YehudAyofF}

\section{References}

[1] P. Balister and B. Bollobás. Projections, entropy and sumsets. Combinatorica, 32:125-141, 2012. $3,5,15$

[2] K. Ball. Volumes and sections of cubes and related problems. In J. Lindenstrauss and V. D. Milman, editors, Geometric Aspects of Functional Analysis, Lecture Notes in Mathematics, Volume 1376, pages 251-260. Springer, Berlin, 1988. 3

[3] K. Ball. Convex geometry and functional analysis. In W. B. Johnson and J. Lindenstrauss, editors, Handbook of the Geometry of Banach Spaces, Volume 1, pages 161-194. Elsevier Science, Amsterdam, 2001. 3

[4] B. Barak, M. Braverman, X. Chen, and A. Rao. How to compress interactive communication. In Proceedings of the 2010 ACM International Symposium on Theory of Computing, pages 67-76, 2010. 5

[5] G. Bianchi and H. Egnell. A note on the Sobolev inequality. Journal of Functional Analysis, 100:18-24, 1991. 3

[6] B. Bollobás and I. Leader. Edge-isoperimetric inequalities in the grid. Combinatorica, 11:299-314, 1991. 6

[7] B. Bollobás and A. Thomason. Projections of bodies and hereditary properties of hypergraphs. Bulletin of the London Mathematical Society, 27:417-424, 1995. 2

[8] H. J. Brascamp and E. H. Lieb. Best constants in Young's inequality, its converse and its generalization to more than three functions. Advances in Mathematics, 20:151-172, 1976. 3, 4

[9] F. R. K. Chung, R. L. Graham, P. Frankl, and J. B. Shearer. Some intersection theorems for ordered sets and graphs. Journal of Combinatorial Theory, Series A, 43(1):23-37, 1986. 2, 3

[10] M. Cicalese and G. P. Leonardi. A selection principle for the sharp quantitative isoperimetric inequality. Archive for Rational Mechanics and Analysis, 206:617-643, 2012. 3

[11] T. M. Cover and J. A. Thomas. Elements of Information Theory. John Wiley \& Sons, New Jersey, 2012. 7

[12] J. Dolbeault and G. Toscani. Improved interpolation inequalities, relative entropy and fast diffusion equations. Annales de l'Institut Henri Poincare (C): Non-Linear Analysis, 30(5):917-934, 2013. 3

[13] D. Ellis. Stability for $t$-intersecting families of permutations. Journal of Combinatorial Theory, Series A, 118:503-528, 2011. 3

[14] D. Ellis, Y. Filmus, and E. Friedgut. A quasi-stability result for dictatorships in $S_{n}$. Combinatorica, 35:573-618, 2015. 3 


\section{GEOMETRIC STABILITY VIA INFORMATION THEORY}

[15] D. Ellis, Y. Filmus, and E. Friedgut. A stability result for balanced dictatorships in $S_{n}$. Random Structures and Algorithms, 46:494-550, 2015. 3

[16] A. Figalli, F. Maggi, and A. Pratelli. A mass transportation approach to quantitative isoperimetric inequalities. Inventiones Mathematicae, 182(1):167-211, 2010. 3

[17] P. Frankl. Erdős-Ko-Rado theorem with conditions on the maximal degree. Journal of Combinatorial Theory, Series A, 46:252-263, 1987. 3

[18] E. Friedgut. Boolean functions with low average sensitivity depend on few coordinates. Combinatorica, 18:27-35, 1998. 3

[19] E. Friedgut. Hypergraphs, entropy and inequalities. The American Mathematical Monthly, 111:749760, 2004. 3, 4

[20] E. Friedgut. On the measure of intersecting families, uniqueness and stability. Combinatorica, 28:503-528, 2008. 3

[21] N. Fusco, F. Maggi, and A. Pratelli. The sharp quantitative isoperimetric inequality. Annals of Mathematics, 168:941-980, 2008. 3, 7

[22] L. H. Loomis and H. Whitney. An inequality related to the isoperimetric inequality. Bulletin of the American Mathematical Society, 55(10):961-962, 1949. 1, 2, 3, 4, 13

[23] F. Maggi. Sets of finite perimeter and geometric variational problems: an introduction to Geometric Measure Theory. Cambridge University Press, Cambridge, 2012. 6

[24] J. Radhakrishnan. Entropy and counting. In J. C. Misra, editor, Computational Mathematics, Modelling and Algorithms. Narosa, New Delhi, India, 2003. 5, 14

[25] R. Raz. A parallel repetition theorem. SIAM Journal on Computing, 27(3):763-803, 1998. 5

[26] M. Simonovits. A method for solving extremal problems in graph theory. In P. Erdős and G. Katona, editors, Theory of Graphs, Proc. Coll. Tihany, 1966, pages 279-319. 1966. 3

\section{AUTHORS}

David Ellis

School of Mathematical Sciences, Queen Mary, University of London, Mile End Road, London E1 4NS, United Kingdom. d.ellis@qmul.ac.uk 


\author{
Ehud Friedgut \\ Department of Mathematics, \\ Weizmann Institute of Science, \\ Rehovot 76100, \\ Israel. \\ ehud.friedgut@gmail.com
}

Guy Kindler

School of Computer Science and Engineering,

The Hebrew University of Jerusalem,

Edmond J. Safra Campus,

Jerusalem 91904,

Israel.

gkindler@cs.huji.ac.il

Amir Yehudayoff

Department of Mathematics,

Technion-IIT,

Haifa 32000,

Israel.

amir.yehudayoff@gmail.com 